\title{
Inequalities for the Lattice Width of Lattice-Free Convex Sets in the Plane
}

\author{
Gennadiy Averkov and Christian Wagner
}

October 9, 2018

\begin{abstract}
A closed, convex set $K$ in $\mathbb{R}^{2}$ with non-empty interior is called lattice-free if the interior of $K$ is disjoint with $\mathbb{Z}^{2}$. In this paper we study the relation between the area and the lattice width of a planar lattice-free convex set in the general and centrally symmetric case. A correspondence between lattice width on the one hand and covering minima on the other, allows us to reformulate our results in terms of covering minima introduced by Kannan and Lovász [12. We obtain a sharp upper bound for the area for any given value of the lattice width. The lattice-free convex sets satisfying the upper bound are characterized. Lower bounds are studied as well. Parts of our results are applied in [1] for cutting plane generation in mixed integer linear optimization, which was the original inducement for this paper. We further rectify a result of [12] with a new proof.
\end{abstract}

Mathematics Subject Classification (AMS 2010): Primary: 52C05; Secondary: 52A38, 52A40, 52C15

Key words and phrases: area; convex set; covering minimum; inhomogeneous minimum; lattice-free body; lattice width

\section{Introduction}

This paper is devoted to the relation between the area and the lattice width (resp. the area and the covering minima) of a lattice-free two-dimensional convex set. Let us denote by $\mathcal{K}^{2}$ the class of closed, convex sets in $\mathbb{R}^{2}$ with non-empty interior. We call a set $K \in \mathcal{K}^{2}$ lattice-free if the interior of $K$ is disjoint with $\mathbb{Z}^{2}$. A convex set $S$ is said to be a strip if $S=\operatorname{conv}\left(l^{1} \cup l^{2}\right)$, where $l^{1}$ and $l^{2}$ are distinct parallel lines. The Euclidean distance between $l^{1}$ and $l^{2}$ is said to be the width of $S$. If the interior of $S$ does not contain integer points and the sets $l^{1} \cap \mathbb{Z}^{2}, l^{2} \cap \mathbb{Z}^{2}$ are affine images of $\mathbb{Z}$, then we call $S$ a split since $S$ splits $\mathbb{Z}^{2}$ into two parts. Let $K \in \mathcal{K}^{2}$. If $S=\operatorname{conv}\left(l^{1} \cup l^{2}\right)$ is a split and $l_{K}^{1}$ and $l_{K}^{2}$ are distinct supporting lines of $K$ parallel to $l^{1}$ and $l^{2}$, we define the strip $S_{K}:=\operatorname{conv}\left(l_{K}^{1} \cup l_{K}^{2}\right)$. Then the lattice width $w(K)$ of $K$ is defined as the minimum of the ratio between the width of $S_{K}$ and the width of $S$ among all splits $S$, for which $S_{K}$ exists. In analytic terms, $w(K):=\min \left\{w(K, u): u \in \mathbb{Z}^{2} \backslash\{o\}\right\}$, where $w(K, u)$ is the width function defined by $w(K, u):=\max \left\{u^{\top} x: x \in K\right\}-\min \left\{u^{\top} x: x \in K\right\}$ for $u \in \mathbb{R}^{2}$.

With these notions our main contribution is the following: for a given lattice-free set $K \in \mathcal{K}^{2}$ we present a list of inequalities which relate its area $A(K)$ to its lattice width $w(K)$, see Theorems 2.2 and 2.4 below. The inequalities which give upper bounds for $A(K)$ for a given $w(K)$, and the sets yielding equality in these inequalities are characterized. For the case of centrally symmetric sets $K \in \mathcal{K}^{2}$ we even give the complete list of inequalities, that is, the lower and upper bounds for $A(K)$ for a given $w(K)$ and a characterization of all pairs $(w(K), A(K))$ where equality is attained. 
All results obtained in this paper can be formulated in terms of an arbitrary lattice, but for the sake of simplicity we use $\mathbb{Z}^{2}$. Let $\Lambda$ be an arbitrary lattice in $\mathbb{R}^{2}$. We can introduce the lattice width $w(K, \Lambda)$ of $K$ with respect to $\Lambda$ (for the precise definition see [11, 12]). Then $w(T(K), T(\Lambda))=w(K, \Lambda)$ for every linear transformation $T$ in $\mathbb{R}^{2}$. Choosing $T$ such that it maps $\Lambda$ onto $\mathbb{Z}^{2}$ we obtain $w(T(K))=$ $w\left(T(K), \mathbb{Z}^{2}\right)=w(T(K), T(\Lambda))=w(K, \Lambda)$. Since $A(T(K)) \cdot \operatorname{det} \Lambda=A(K)$, every inequality involving the area and the lattice width with respect to $\mathbb{Z}^{2}$ can be transformed to an inequality involving the area, the lattice width with respect to $\Lambda$ and $\operatorname{det} \Lambda$. For figures it is sometimes more convenient to use the lattice of regular triangles, i.e., the lattice generated by the vectors $(1,0)$ and $\left(\frac{1}{2}, \frac{\sqrt{3}}{2}\right)$. For information on lattices and convexity we refer to 7 and 8 .

Our motivation for studying these relations was the application of our results for a classification of three-dimensional lattice-free polyhedra having an integer point in the relative interior of each facet, see [1. Such class of polyhedra can be used in mixed integer linear programming for deriving cutting planes; see also [18, Chapter 23] for background information on cutting plane theory.

The second covering minimum $\mu_{2}(K)$ of $K \in \mathcal{K}^{2}$ is defined as the minimal $t \geq 0$ such that the sets $t K+\mathbb{Z}^{2}$ cover $\mathbb{R}^{2}$. The value $\mu_{2}(K)$ is also known under the name of inhomogeneous minimum, see 8 , p. 98]. It turns out that some translate of $K$ is lattice-free if and only if $\mu_{2}(K) \geq 1$. The first covering minimum $\mu_{1}(K)$ is defined as the minimal $t \geq 0$ such that every line in $\mathbb{R}^{2}$ intersects $t K+\mathbb{Z}^{2}$. One can show that $\mu_{1}(K) w(K)=1$ for every $K \in \mathcal{K}^{2}$, see e.g. [12, Lemma 2.3]. This leads to a correspondence between the lattice width on the one hand and the covering minima on the other, provided that $K$ is lattice-free. The results we present in this paper can therefore be expressed as a relation between the area and the covering minima of $K$, as well; see Corollaries 2.6 and 2.7. The notions lattice width and covering minima were introduced by Kannan and Lovász [11, 12, for an arbitrary dimension; see also the papers of Khinchin [13] and Fejes Tóth and Makai Jr. 6] for the earlier related results.

The paper has the following structure. Our main results are stated in Section 2, Basic notions and results which we shall need to prove our theorems are introduced in Section 3 . Section 4 presents formulas for the lattice width and area of triangles. Section 5 contains the proofs for general planar lattice-free convex sets. The proofs for the centrally symmetric planar lattice-free convex sets are given in Section 6 .

\section{Results}

Throughout the paper, sequences with $n$ elements are indexed modulo $n$. Affine transformations preserving $\mathbb{Z}^{2}$ will be called unimodular.

For the plane it is easy to construct examples of lattice-free convex sets with a lattice width of two; take for example conv $\{(0,0),(2,0),(0,2)\}$. What may be surprising, this value can be exceeded. This was noticed by Hurkens [10, who also computed the sharp upper bound for the lattice width of a lattice-free convex set.

Theorem 2.1. [10, p. 122] Let $K \in \mathcal{K}^{2}$ be lattice-free. Then

$$
w(K) \leq 1+\frac{2}{\sqrt{3}}
$$

with equality if and only if $K$ is a triangle with vertices $q_{0}, q_{1}, q_{2}$ such that, for every $i$, the point $p_{i}:=$ $\frac{1}{\sqrt{3}} q_{i+1}+\left(1-\frac{1}{\sqrt{3}}\right) q_{i+2}$ belongs to $\mathbb{Z}^{2}$ (see Fig. 1).

Theorem 2.2 states the relation between the area and the lattice width of arbitrary lattice-free convex sets in the plane. 


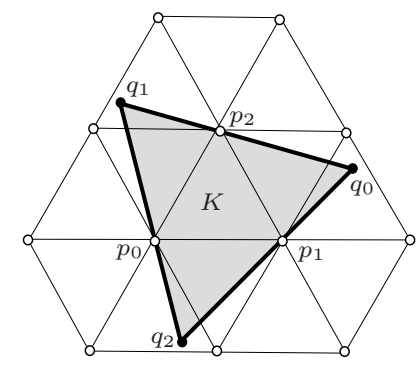

Figure 1: Lattice-free triangle (shaded) with lattice width $1+\frac{2}{\sqrt{3}}$

Theorem 2.2. Let $K \in \mathcal{K}^{2}$ be lattice-free with $w:=w(K)$ and $A:=A(K)$. Then

$$
\begin{aligned}
& A \leq \infty \quad \text { for } \quad 0<w \leq 1, \\
& A \leq \frac{w^{2}}{2(w-1)} \quad \text { for } \quad 1<w \leq 2, \\
& A \leq \frac{3 w^{2}}{3 w+1-\sqrt{1+6 w-3 w^{2}}} \quad \text { for } \quad 2<w \leq 1+\frac{2}{\sqrt{3}} \text {, } \\
& A \geq \frac{3}{8} w^{2} \quad \text { for } \quad 0<w \leq 1+\frac{2}{\sqrt{3}}
\end{aligned}
$$

(see Fig. 3). Furthermore, the following statements hold.

I. Equality in (2.2) is attained if and only if $K$ is unbounded and contained in a split.

II. Equality in (2.3) is attained if and only if, up to unimodular transformations, $K=\operatorname{conv}\left(I_{1} \cup I_{2}\right)$, where $I_{1}$ is a translate of $\operatorname{conv}\{(0,0),(w, 0)\}, I_{2}$ is a translate of $\operatorname{conv}\left\{(0,0),\left(0, \frac{w}{w-1}\right)\right\}$, and $I_{1} \cap I_{2} \neq$ $\emptyset$ (see Fig. 2(a)).

III. Equality in (2.4) is attained if and only if $K$ is a triangle with vertices $q_{0}, q_{1}, q_{2}$ such that, for every $i$, the point $p_{i}:=\lambda q_{i+1}+(1-\lambda) q_{i+2}$ belongs to $\mathbb{Z}^{2}$ for

$$
\lambda:=\frac{3 w+1-\sqrt{1+6 w-3 w^{2}}}{6 w}
$$

(see Fig. 2(b)).

$I V$. If $0<w \leq 2$, then equality in (2.5) is attained if and only if, up to unimodular transformations, $K$ is a translate of $\frac{w}{2} \operatorname{conv}\{(1,0),(0,1),(-1,-1)\}$ (see Fig. 2(c)).

The bound (2.5) is not sharp when $2<w \leq 1+\frac{2}{\sqrt{3}}$. To see why, we need a result of Fejes Tóth and Makai Jr. 6].

Theorem 2.3. 6] Let $K \in \mathcal{K}^{2}$ with $w:=w(K)$ and $A:=A(K)$. Then $A \geq \frac{3}{8} w^{2}$ with equality if and only if, up to unimodular transformations, $K$ is a translate of $\frac{w}{2} \operatorname{conv}\{(1,0),(0,1),(-1,-1)\}$. 


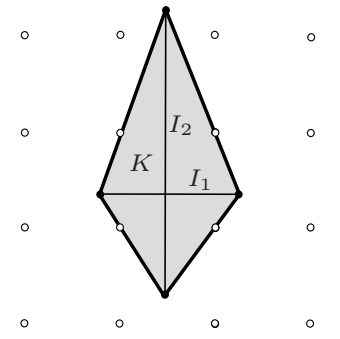

(a) Illustration to 2.3 and Part II

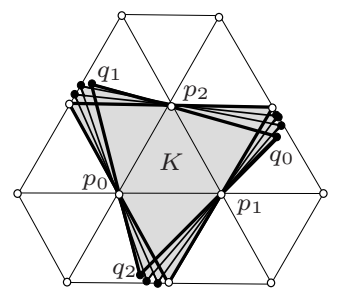

(b) Illustration to 2.4 and Part [II

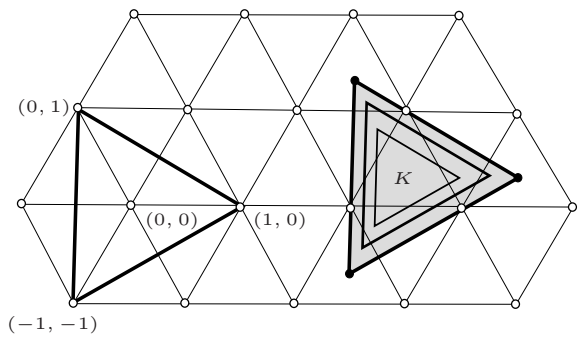

(c) Illustration to 2.5 and Part IV

Figure 2: Examples of sets yielding equality in (2.3)-(2.5) (shaded)

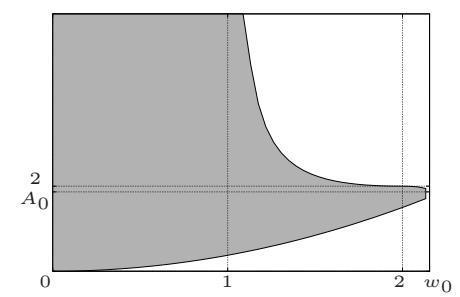

Figure 3: Pairs $(w, A)$ satisfying the inequalities of Theorem 2.2, $w_{0}=1+2 / \sqrt{3}$, $A_{0}$ is the area of $K$ with $w(K)=w_{0}$

It is easy to see that $\frac{w}{2} \operatorname{conv}\{(1,0),(0,1),(-1,-1)\}$ does not have a lattice-free translate for $w>2$. Thus, in view of Theorem 2.3, (2.5) is not sharp when $2<w \leq 1+\frac{2}{\sqrt{3}}$. The problem to find the sharp lower bound in this case is still open.

A statement analogous to Theorem 2.2 can also be proved for the class of centrally symmetric planar convex sets. We show the following theorem.

Theorem 2.4. Let $K \in \mathcal{K}^{2}$ be lattice-free and centrally symmetric with $w:=w(K)$ and $A:=A(K)$. Then

$$
\begin{aligned}
& 0<w \leq 2 \\
& A \leq \infty \quad \text { for } \quad 0<w \leq 1, \\
& A \leq \frac{w^{2}}{2(w-1)} \quad \text { for } \quad 1<w \leq 2, \\
& A \geq \frac{1}{2} w^{2} \quad \text { for } \quad 0<w \leq 2
\end{aligned}
$$

(see Fig. 國). Furthermore, the following statements hold.

I. The upper bound in (2.6) is attained if and only if, up to unimodular transformations,

$$
K=\operatorname{conv}\{ \pm(1,0), \pm(0,1)\}+\left(\frac{1}{2}, \frac{1}{2}\right) .
$$


II. Equality in (2.7) is attained if and only if $K$ is unbounded and contained in a split.

III. Equality in (2.8) is attained if and only if, up to unimodular transformations,

$$
K=\operatorname{conv}\left\{ \pm\left(\frac{w}{2}, 0\right), \pm\left(0, \frac{w}{2(w-1)}\right)\right\}+\left(\frac{1}{2}, \frac{1}{2}\right)
$$

(see Fig. 4(a)).

$I V$. Equality in (2.9) is attained if and only if, up to unimodular transformations, $K$ is a translate of

$$
\frac{w}{2} \operatorname{conv}\{ \pm(1, \alpha), \pm(0,1)\}
$$

for some $0 \leq \alpha<1$ satisfying $\max \{1+\alpha, 2-\alpha\} \geq w$ (see Fig. 4(b)).

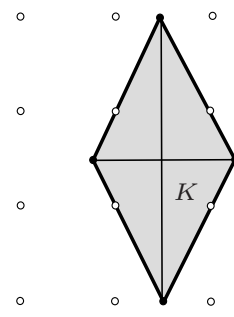

(a) Illustration to 2.8 and Part

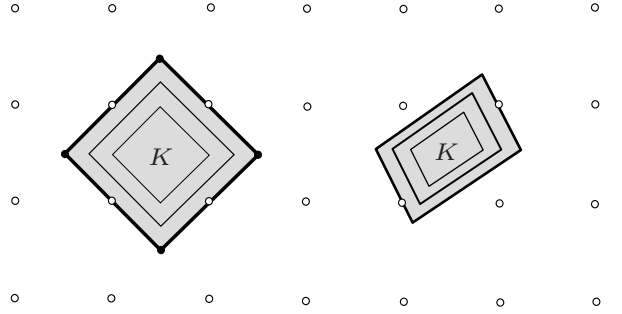

(b) Illustration to 2.9) and Part IV (with examples of $K$ for two different values of $\alpha$ )

Figure 4: Examples of sets yielding equality in (2.8) and (2.9) (shaded)

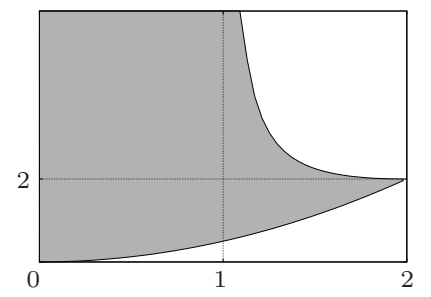

Figure 5: All pairs $(w, A)$ satisfying the inequalities of Theorem 2.4

A useful tool in convex geometry is the concept of covering minima introduced by Kannan and Lovász 12. We will therefore give an alternative formulation of our results in terms of covering minima. Let $K \in \mathcal{K}^{2}$. For $j=1,2$ the $j$-th covering minimum is

$$
\mu_{j}(K):=\inf \left\{t \geq 0: \text { each }(2-j) \text {-dimensional affine subspace of } \mathbb{R}^{2} \text { intersects } t K+\mathbb{Z}^{2}\right\} .
$$

This definition implies $0<\mu_{1} \leq \mu_{2}$. We recall that $\mu_{1}(K) w(K)=1$. Furthermore, for $t>0$, an appropriate translate of $t K$ is lattice-free if and only if $t \leq \mu_{2}(K)$. Thus, Theorem 2.1 yields $\mu_{2}(K) \leq$ $\left(1+\frac{2}{\sqrt{3}}\right) \mu_{1}(K)$. 
For centrally symmetric bodies, the lower bound in (2.9) was noticed by Makai Jr. 16. The upper bound in (2.6) is a consequence of a more general result due to Kannan and Lovász [12, Theorem 2.13]. Unfortunately, the proof of Theorem 2.13 in [12] does not seem to be correct1], but implies the weaker result $0<w \leq 3$. Therefore, we prove the subsequent theorem.

Theorem 2.5. Let $K \in \mathcal{K}^{2}$ be centrally symmetric. Then $\mu_{2}(K) \leq 2 \mu_{1}(K)$.

If $K$ is lattice-free, and thus $\mu_{2}(K) \geq 1$, then together with the relation $\mu_{1}(K) w(K)=1$, Theorem 2.5 implies $w(K) \leq 2$. The results stated in Theorems 2.2 and 2.4 can also be expressed in terms of covering minima. In Corollary 2.6 the lower bound for $A(K)$ in terms of $\mu_{1}(K)$ and $\mu_{2}(K)$ goes back to 6] (see also 3 for further inequalities). Our results imply the sharp upper bounds for $A(K)$ in terms of $\mu_{1}(K)$ and $\mu_{2}(K)$.

Corollary 2.6. Let $K \in \mathcal{K}^{2}$ with $A:=A(K), \mu_{1}:=\mu_{1}(K)$ and $\mu_{2}:=\mu_{2}(K)$. Then

$$
\begin{aligned}
& \mu_{1} \leq \mu_{2} \leq\left(1+\frac{2}{\sqrt{3}}\right) \mu_{1}, \\
& A \leq \infty \quad \text { for } \quad \mu_{1}=\mu_{2}, \\
& A \leq \frac{1}{2 \mu_{1}\left(\mu_{2}-\mu_{1}\right)} \quad \text { for } \quad \mu_{1}<\mu_{2} \leq 2 \mu_{1} \text {, } \\
& A \leq \frac{3}{\mu_{1}\left(3 \mu_{2}+\mu_{1}-\sqrt{\mu_{1}^{2}+6 \mu_{1} \mu_{2}-3 \mu_{2}^{2}}\right)} \quad \text { for } \quad 2 \mu_{1}<\mu_{2} \leq\left(1+\frac{2}{\sqrt{3}}\right) \mu_{1}, \\
& A \geq \frac{3}{8 \mu_{1}^{2}} \quad \text { for } \quad \mu_{1} \leq \mu_{2} \leq\left(1+\frac{2}{\sqrt{3}}\right) \mu_{1} \text {. }
\end{aligned}
$$

The upper bounds for $A$ are sharp, whereas the lower bound for $A$ is sharp only for $\mu_{1} \leq \mu_{2} \leq 2 \mu_{1}$.

Corollary 2.7. Let $K \in \mathcal{K}^{2}$ be centrally symmetric with $A:=A(K), \mu_{1}:=\mu_{1}(K)$ and $\mu_{2}:=\mu_{2}(K)$. Then

$$
\begin{aligned}
\mu_{1} & \leq \mu_{2} \leq 2 \mu_{1}, & & \\
A & \leq \infty & \text { for } & \mu_{1}=\mu_{2}, \\
A & \leq \frac{1}{2 \mu_{1}\left(\mu_{2}-\mu_{1}\right)} & \text { for } & \mu_{1}<\mu_{2} \leq 2 \mu_{1}, \\
A & \geq \frac{1}{2 \mu_{1}^{2}} & \text { for } & \mu_{1} \leq \mu_{2} \leq 2 \mu_{1} .
\end{aligned}
$$

The above bounds are sharp.

For further inequalities between $\mu_{1}(K), \mu_{2}(K)$ and $A(K)$ for the case $K \in \mathcal{K}^{2}$ we refer to [17].

\footnotetext{
${ }^{1}$ In the proof of Theorem 2.13 in [12] one claims that the covering minima of centrally symmetric convex bodies satisfy the inequalities $\mu_{k+1} \leq 2 \mu_{k}$ (see 12 for the explanation of the notations). In the proof of Theorem 2.13, p. 588, 1. 11, it is inferred that $2\left(\alpha+\beta+\lambda_{1}\right) \leq 4(\alpha+\beta)$. However, one line before it is just shown that $\lambda_{1} \leq 2(\alpha+\beta)$ and therefore the correct conclusion is $2\left(\alpha+\beta+\lambda_{1}\right) \leq 6(\alpha+\beta)$. Using the factor 6 instead of 4 in the rest of the proof results in the weaker assertion $\mu_{k+1} \leq 3 \mu_{k}$. To the best of our knowledge there is no revision or corrected version of this proof which would yield the assertion $\mu_{k+1} \leq 2 \mu_{k}$.
} 


\section{Preliminaries}

We consider the elements of $\mathbb{R}^{2}$ to be column vectors. The transposition is denoted by $(\cdot)^{\top}$ and the origin by $o$. By aff, conv, bd and int we denote the affine hull, the convex hull, the boundary and the interior, respectively. The maximum norm is denoted by $\|\cdot\|_{\infty}$. Remember that a set $K \in \mathcal{K}^{2}$ is said to be lattice-free if the interior of $K$ is disjoint with $\mathbb{Z}^{2}$. A lattice-free set $K \in \mathcal{K}^{2}$ is said to be maximal lattice-free if $K$ is not properly contained in a lattice-free set from $\mathcal{K}^{2}$. The following fact is known.

Proposition 3.1. Every lattice-free $K \in \mathcal{K}^{2}$ is contained in a maximal lattice-free $H \in \mathcal{K}^{2}$.

Proof. For a closed convex set $U \subseteq \mathbb{R}^{2}$ and a point $x \in \mathbb{R}^{2}$ we denote by $c(U, x)$ the topological closure of the convex hull of $U \cup\{x\}$.

Let $\left(z_{n}\right)_{n=1}^{\infty}$ be the sequence of all elements of $\mathbb{Q}^{2}$. We define $U_{0}:=K$ and for every $n \in \mathbb{N}$ we set $U_{n}:=c\left(U_{n-1}, z_{n}\right)$ if $c\left(U_{n-1}, z_{n}\right)$ is lattice-free, and $U_{n}:=U_{n-1}$ otherwise. Let $H$ be the topological closure of $\bigcup_{n=0}^{\infty} U_{n}$. Since $U_{n-1} \subseteq U_{n}$ for every $n \in \mathbb{N}$ it holds $K \subseteq H$. By construction, $H$ is a closed convex set with non-empty interior, i.e., $H \in \mathcal{K}^{2}$. In addition, $H$ is lattice-free: assume $y$ is an integer point in the interior of $H$. Then there exists some $j \in \mathbb{N}$ such that $y$ is in the interior of $U_{j}$ and thus, $U_{j}$ is not lattice-free. This contradicts the construction of $U_{j}$. Let us show that $H$ is maximal lattice-free. Assume the opposite and let $L \in \mathcal{K}^{2}$ be lattice-free such that $H \varsubsetneqq L$. Then $L \backslash H$ contains rational points which occur in the sequence $\left(z_{n}\right)_{n=1}^{\infty}$. Let $z_{k} \in L \backslash H$ be such a rational point. Since $U_{k-1} \subseteq H \subseteq L$ and $z_{k} \in L$, we have $c\left(U_{k-1}, z_{k}\right) \subseteq L$. Thus, $c\left(U_{k-1}, z_{k}\right)$ is lattice-free and, by definition of $U_{k}$, we have $U_{k}=c\left(U_{k-1}, z_{k}\right)$. The latter implies $z_{k} \in H$, a contradiction to the choice of $z_{k}$.

We point out that the above proof is not constructive. For a constructive, but lengthy proof we refer to 2 .

A classification of planar maximal lattice-free convex sets was noticed by Lovász 14. The refined classification which is given below can be found in [5].

Proposition 3.2. [5] 14 Let $K \in \mathcal{K}^{2}$ be maximal lattice-free. Then $K$ is either a split or a triangle or a quadrilateral and the relative interior of each facet of $K$ contains at least one integer point. In particular, $K$ is one of the following sets (see Fig. (6).

I. A split $\left\{\left(x_{1}, x_{2}\right) \in \mathbb{R}^{2}: b \leq a_{1} x_{1}+a_{2} x_{2} \leq b+1\right\}$ where $a_{1}$ and $a_{2}$ are coprime integers and $b$ is an integer.

II. A triangle with at least one integer point in the relative interior of each of its edges, which in turn is either

(a) a type 1 triangle, i.e., a triangle with integer vertices and exactly one integer point in the relative interior of each edge, or

(b) a type 2 triangle, i.e., a triangle with at least one fractional vertex $v$, exactly one integer point in the relative interior of the two edges incident to $v$ and at least two integer points on the third edge, or

(c) a type 3 triangle, i.e., a triangle with exactly three integer points on the boundary, one in the relative interior of each edge.

III. A quadrilateral containing exactly one integer point in the relative interior of each of its edges. 


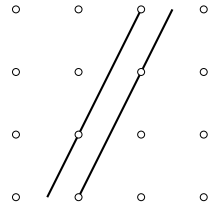

(a) Split

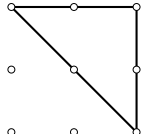

(b) Type 1 triangle

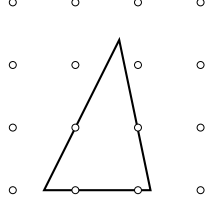

(c) Type 2 triangle

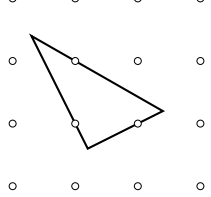

(d) Type 3 triangle

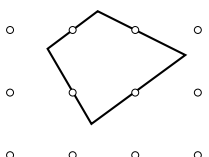

(e) Quadrilateral

Figure 6: All types of maximal lattice-free sets in $\mathcal{K}^{2}$

Theorems 2.2 and 2.4 will be proved for maximal lattice-free convex sets first. Once this is established we show that this implies their validity for lattice-free convex sets which are not maximal as well.

For $K \in \mathcal{K}^{2}$, the support function of $K$ is defined by $h(K, u):=\sup \left\{u^{\top} x: x \in K\right\}$. Remember that the width function of $K$ is $w(K, u)=h(K, u)+h(K,-u)$, where $u \in \mathbb{R}^{2}$, and the lattice width of $K$ is the value $w(K)=\min \left\{w(K, u): u \in \mathbb{Z}^{2} \backslash\{o\}\right\}$. Note that the lattice width is invariant with respect to unimodular transformations. Bounded elements of $\mathcal{K}^{2}$ are referred to as convex bodies. If $K \in \mathcal{K}^{2}$ is a convex body, then $D K:=\{x-y: x, y \in K\}$ is said to be the difference body of $K$. It is well known that $h(D K, u)=w(K, u)$ for every $u \in \mathbb{R}^{2}$. If $K$ is a convex body symmetric in the origin, then the Minkowski functional $\|\cdot\|_{K}$ of $K$ is defined by $\|u\|_{K}:=\min \{\lambda \geq 0: u \in \lambda K\}$, where $u \in \mathbb{R}^{2}$. For a convex body $K$ containing the origin in the interior the set $K^{*}:=\left\{u \in \mathbb{R}^{2}: h(K, u) \leq 1\right\}$ is a convex body as well and is referred to as the polar body of $K$. One has $h(K, u)=\|u\|_{K^{*}}$ for every $u \in \mathbb{R}^{2}$. The following formula, in slightly different terms, can be found in [12, Lemma 2.3]:

$$
w(K)=\sup \left\{\alpha>0:\left(\alpha(D K)^{*}\right) \cap \mathbb{Z}^{2}=\{o\}\right\} .
$$

A collection of convex sets $\mathcal{X}$ from $\mathcal{K}^{2}$ is said to tile $\mathbb{R}^{2}$ if the interiors of the elements of $\mathcal{X}$ are pairwise disjoint and the union of $\mathcal{X}$ yields $\mathbb{R}^{2}$ (see also [19, Section 4.1] for information on lattice tilings).

The two-dimensional version of Minkowski's first fundamental theorem (see [7, Theorem 22.1]) states that if $K \in \mathcal{K}^{2}$ is symmetric in the origin and int $K \cap \mathbb{Z}^{2}=\{o\}$, then $A(K) \leq 4$. Furthermore, if $A(K)=4$, then the sets $\frac{1}{2} K+z$ with $z \in \mathbb{Z}^{2}$ tile $\mathbb{R}^{2}$. Mahler's inequality (see [15]) states that $A(K) A\left(K^{*}\right) \geq 8$ for convex bodies $K \in \mathcal{K}^{2}$ symmetric in the origin, with equality if and only if $K$ is a parallelogram. The following proposition is easy to show.

Proposition 3.3. Let $P$ be a parallelogram symmetric in the origin and such that its translates $P+z$ with $z \in \mathbb{Z}^{2}$ tile $\mathbb{R}^{2}$. Then, up to unimodular transformations, $P=\frac{1}{2} \operatorname{conv}\{ \pm(-\alpha-1,1), \pm(-\alpha+1,1)\}$ for some $0 \leq \alpha<1$.

Proposition 3.3 is a special case of a result of Hajós [9, $\S \S 1,2]$, see also [8, p. 174].

\section{Auxiliary results on triangles}

An essential part of the proofs of our main results is concerned with analytical representations of the lattice width and the area of various types of maximal lattice-free polygons. In particular, we shall need formulas for the lattice width of triangles.

We start the section by presenting well-known facts about barycentric coordinates. 
Lemma 4.1. Let $q_{0}, q_{1}, q_{2}$ be affinely independent points in $\mathbb{R}^{2}$ and let $p, p_{0}, p_{1}, p_{2}$ be points in $\mathbb{R}^{2}$ which are represented in the form

$$
p=\sum_{j=0}^{2} x_{j} q_{j} \quad \text { and } \quad p_{i}=\sum_{j=0}^{2} x_{i, j} q_{j},
$$

where

$$
1=\sum_{j=0}^{2} x_{j} \quad \text { and } \quad 1=\sum_{j=0}^{2} x_{i, j},
$$

and $x_{j}, x_{i, j} \in \mathbb{R}$ for $i, j=0,1,2$. We define $l_{j}:=\operatorname{aff}\left(\left\{q_{0}, q_{1}, q_{2}\right\} \backslash\left\{q_{j}\right\}\right)$ for $j=0,1,2$. Then the following statements hold.

I. The points $p$ and $q_{j}$ lie in the same open halfplane determined by $l_{j}$ if and only if $x_{j}>0$.

II. The value $\left|x_{j}\right|$ is the ratio of the distance from $p$ to $l_{j}$ and the distance from $q_{j}$ to $l_{j}$.

III. The areas of $Q:=\operatorname{conv}\left\{q_{0}, q_{1}, q_{2}\right\}$ and $P:=\operatorname{conv}\left\{p_{0}, p_{1}, p_{2}\right\}$ are related by

$$
A(P)=\left|\operatorname{det}\left(x_{i, j}\right)_{i, j=0, \ldots, 2}\right| A(Q) .
$$

The values $x_{0}, x_{1}, x_{2}$ associated to a point $p$ in Lemma 4.1 are said to be the barycentric coordinates of $p$ with respect to the triangle $\operatorname{conv}\left\{q_{0}, q_{1}, q_{2}\right\}$, see [4, Sect. 13.7].

Lemma 4.2. Let $P:=\operatorname{conv}\left\{p_{0}, p_{1}, p_{2}\right\}$ such that $p_{0}, p_{1}, p_{2} \in \mathbb{Z}^{2}$ are the only integer points in $P$. Let $Q:=\operatorname{conv}\left\{q_{0}, q_{1}, q_{2}\right\}$ be a triangle whose vertices are given by the barycentric coordinates with respect to $P$, that is, by a $3 \times 3$-matrix $B$ such that

$$
\left(\begin{array}{ll}
q_{0}^{\top} & 1 \\
q_{1}^{\top} & 1 \\
q_{2}^{\top} & 1
\end{array}\right)=B\left(\begin{array}{ll}
p_{0}^{\top} & 1 \\
p_{1}^{\top} & 1 \\
p_{2}^{\top} & 1
\end{array}\right)
$$

Then

$$
w(Q)=\min \left\{\|D B z\|_{\infty}: z \in \mathbb{Z}^{3} \text { and the coordinates of } z \text { are not all equal }\right\}
$$

where

$$
D:=\left(\begin{array}{rrr}
-1 & 1 & 0 \\
0 & -1 & 1 \\
1 & 0 & -1
\end{array}\right)
$$

Furthermore, if $p_{i}:=\left(1-x_{i}\right) q_{i+1}+x_{i} q_{i+2}$ with $i=0,1,2$ and $0<x_{i}<1$ (that is, $Q$ is circumscribed about $P$ ), then

$$
w(Q)=\frac{\min \left\{\max _{i=0,1,2}\left|x_{i} y_{i}+\left(1-x_{i+1}\right) y_{i+1}\right|: y \in \mathbb{Z}^{3} \backslash\{o\}, y_{0}+y_{1}+y_{2}=0\right\}}{x_{0} x_{1} x_{2}+\left(1-x_{0}\right)\left(1-x_{1}\right)\left(1-x_{2}\right)}
$$

and

$$
A(Q)=\frac{1}{2\left(x_{0} x_{1} x_{2}+\left(1-x_{0}\right)\left(1-x_{1}\right)\left(1-x_{2}\right)\right)} .
$$


Proof. For $u \in \mathbb{Z}^{2}$ we have

$$
\begin{aligned}
w(Q, u) & =\max \left\{\left|q_{i}^{\top} u-q_{j}^{\top} u\right|: 0 \leq i<j \leq 2\right\}=\left\|D\left(\begin{array}{l}
q_{0}^{\top} \\
q_{1}^{\top} \\
q_{2}^{\top}
\end{array}\right) u\right\|_{\infty}=\left\|D\left(\begin{array}{ll}
q_{0}^{\top} & 1 \\
q_{1}^{\top} & 1 \\
q_{2}^{\top} & 1
\end{array}\right)\left(\begin{array}{l}
u \\
k
\end{array}\right)\right\|_{\infty} \\
& =\left\|D B\left(\begin{array}{ll}
p_{0}^{\top} & 1 \\
p_{1}^{\top} & 1 \\
p_{2}^{\top} & 1
\end{array}\right)\left(\begin{array}{l}
u \\
k
\end{array}\right)\right\|_{\infty}=\|D B z\|_{\infty},
\end{aligned}
$$

where $k \in \mathbb{Z}$ is arbitrary and

$$
z:=\left(\begin{array}{ll}
p_{0}^{\top} & 1 \\
p_{1}^{\top} & 1 \\
p_{2}^{\top} & 1
\end{array}\right)\left(\begin{array}{l}
u \\
k
\end{array}\right)=\left(\begin{array}{l}
p_{0}^{\top} u+k \\
p_{1}^{\top} u+k \\
p_{2}^{\top} u+k
\end{array}\right)
$$

Clearly, $z \in \mathbb{Z}^{3}$. Since the vector $z$ is the product of a unimodular matrix and an integer vector, it follows that $u=o$ if and only if the coordinates of $z$ are all equal. This shows (4.1).

Let us show (4.2). We have

$$
\left(\begin{array}{ll}
p_{0}^{\top} & 1 \\
p_{1}^{\top} & 1 \\
p_{2}^{\top} & 1
\end{array}\right)=X\left(\begin{array}{ll}
q_{0}^{\top} & 1 \\
q_{1}^{\top} & 1 \\
q_{2}^{\top} & 1
\end{array}\right), \quad \text { where } \quad X:=B^{-1}=\left(\begin{array}{ccc}
0 & 1-x_{0} & x_{0} \\
x_{1} & 0 & 1-x_{1} \\
1-x_{2} & x_{2} & 0
\end{array}\right) .
$$

Since $X$ is the matrix of barycentric coordinates of the vertices of $P$ with respect to $Q, B$ is the matrix of barycentric coordinates of the vertices of $Q$ with respect to $P$. Direct computations yield

$$
\begin{aligned}
\operatorname{det} X & =x_{0} x_{1} x_{2}+\left(1-x_{0}\right)\left(1-x_{1}\right)\left(1-x_{2}\right)>0 \\
B & =\frac{1}{\operatorname{det} X}\left(\begin{array}{ccc}
-\left(1-x_{1}\right) x_{2} & x_{0} x_{2} & \left(1-x_{0}\right)\left(1-x_{1}\right) \\
\left(1-x_{1}\right)\left(1-x_{2}\right) & -\left(1-x_{2}\right) x_{0} & x_{0} x_{1} \\
x_{1} x_{2} & \left(1-x_{0}\right)\left(1-x_{2}\right) & -\left(1-x_{0}\right) x_{1}
\end{array}\right), \\
D B & =\frac{1}{\operatorname{det} X}\left(\begin{array}{ccc}
1-x_{1} & -x_{0} & x_{0}+x_{1}-1 \\
x_{1}+x_{2}-1 & 1-x_{2} & -x_{1} \\
-x_{2} & x_{0}+x_{2}-1 & 1-x_{0}
\end{array}\right) .
\end{aligned}
$$

We employ the latter matrix relation and obtain for every $z \in \mathbb{Z}^{3}$ the following:

$$
D B z=\frac{1}{\operatorname{det} X}\left(\begin{array}{cccc}
z_{2}-z_{1} & z_{2}-z_{0} & 0 & z_{0}-z_{2} \\
0 & z_{0}-z_{2} & z_{0}-z_{1} & z_{1}-z_{0} \\
z_{1}-z_{2} & 0 & z_{1}-z_{0} & z_{2}-z_{1}
\end{array}\right)\left(\begin{array}{c}
x_{0} \\
x_{1} \\
x_{2} \\
1
\end{array}\right)
$$

By the change of variables

$$
y:=\left(\begin{array}{c}
z_{2}-z_{1} \\
z_{0}-z_{2} \\
z_{1}-z_{0}
\end{array}\right)
$$


the latter amounts to

$$
\begin{aligned}
D B z & =\frac{1}{\operatorname{det} X}\left(\begin{array}{rrrr}
y_{0} & -y_{1} & 0 & y_{1} \\
0 & y_{1} & -y_{2} & y_{2} \\
-y_{0} & 0 & y_{2} & y_{0}
\end{array}\right)\left(\begin{array}{c}
x_{0} \\
x_{1} \\
x_{2} \\
1
\end{array}\right) \\
& =\frac{1}{\operatorname{det} X}\left(\begin{array}{l}
x_{0} y_{0}+\left(1-x_{1}\right) y_{1} \\
x_{1} y_{1}+\left(1-x_{2}\right) y_{2} \\
x_{2} y_{2}+\left(1-x_{0}\right) y_{0}
\end{array}\right)
\end{aligned}
$$

Clearly, $y_{0}+y_{1}+y_{2}=0$ and, if the coordinates of $z$ are not all equal, we have $y \neq o$. Conversely, for an arbitrary $y \in \mathbb{Z}^{3} \backslash\{o\}$ with $y_{0}+y_{1}+y_{2}=0$, we can easily find an appropriate $z \in \mathbb{Z}^{3}$ satisfying (4.4). Thus, employing (4.1) and the previous derivation we arrive at

$$
w(Q)=\frac{1}{\operatorname{det} X} \min \left\{\max \left\{\left|x_{i} y_{i}+\left(1-x_{i+1}\right) y_{i+1}\right|: i=0,1,2\right\}: y \in \mathbb{Z}^{3} \backslash\{o\}, y_{0}+y_{1}+y_{2}=0\right\} .
$$

Equation (4.3) is a straightforward consequence of Lemma 4.1III]

\section{$5 \quad$ Proofs for arbitrary bodies}

In order to prove Theorem 2.2 it is convenient to show the statement only for maximal lattice-free convex sets, instead of lattice-free ones. This is possible since every lattice-free convex set is contained in a maximal lattice-free convex set, see Proposition 3.1

Lemma 5.1 characterizes, in analytic terms, maximal lattice-free triangles of type 3 and their lattice width.

Lemma 5.1. Let $P:=\operatorname{conv}\left\{p_{0}, p_{1}, p_{2}\right\}$ such that $p_{0}, p_{1}, p_{2} \in \mathbb{Z}^{2}$ are the only integer points in $P$. Let $Q:=\operatorname{conv}\left\{q_{0}, q_{1}, q_{2}\right\}$ be a triangle circumscribed about $P$ so that $p_{i}:=\left(1-x_{i}\right) q_{i+1}+x_{i} q_{i+2}$ for $i=0,1,2$ and $0<x_{i}<1$ (see also Fig. (7). Then the following statements hold.

I. $Q$ is a maximal lattice-free triangle of type 3 if and only if

(a) $x_{i}+x_{j}>1$ for all $0 \leq i<j \leq 2$ or

(b) $x_{i}+x_{j}<1$ for all $0 \leq i<j \leq 2$.

II. If (a) holds, then the lattice width of $Q$ is given by

$$
w(Q)=\frac{\min \left\{x_{0}, x_{1}, x_{2}\right\}}{x_{0} x_{1} x_{2}+\left(1-x_{0}\right)\left(1-x_{1}\right)\left(1-x_{2}\right)} .
$$

III. If $(a)$ holds, then $w(Q) \leq 1+\frac{2}{\sqrt{3}}$ with equality if and only if $x_{0}=x_{1}=x_{2}=\frac{1}{\sqrt{3}}$.

Proof. Assume that $Q$ is a lattice-free triangle of type 3. By $H_{i}$ we denote the closed halfplane with $q_{i+1}, q_{i+2} \in \operatorname{bd} H_{i}$ and $q_{i} \in H_{i}$. We also introduce the points $r_{i}:=-p_{i}+p_{i+1}+p_{i+2} \in \mathbb{Z}^{2}$. By construction, $p_{i}$ is the midpoint of $\operatorname{conv}\left\{r_{i+1}, r_{i+2}\right\}$. Because of the latter property, and since $p_{i} \in \operatorname{bd} H_{i}$, we have $r_{i+1} \in H_{i}$ or $r_{i+2} \in H_{i}$. For $i \in\{0,1,2\}$ by $\tau(i)$ we denote the set of all $k \in\{0,1,2\}$ such that $k \neq i$ and $r_{k} \in H_{i}$. By the above observations $\tau(i) \neq \emptyset$ for every $i$. If for some $0 \leq i<j \leq 2$ one has $\tau(i) \cap \tau(j) \neq \emptyset$ we choose $k \in \tau(i) \cap \tau(j)$. Then $r_{k} \in H_{0} \cap H_{1} \cap H_{2}=Q$, and by this the cell 


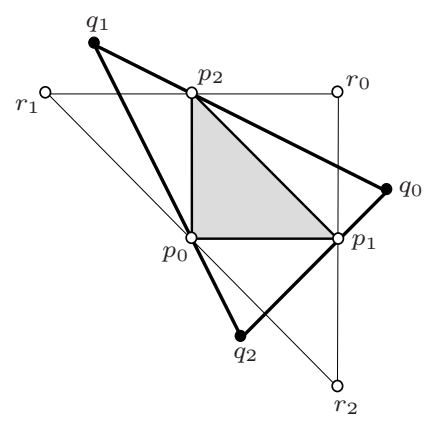

Figure 7: Points $p_{i}, q_{i}, r_{i}, i \in\{0,1,2\}$, as in the proof of Lemma 5.1

$\operatorname{conv}\left\{p_{0}, p_{1}, p_{2}, r_{k}\right\}$ of $\mathbb{Z}^{2}$ is a subset of $Q$. The latter means that $Q$ is not of type 3 , a contradiction. Thus, $\tau(i) \cap \tau(j)=\emptyset$ for $0 \leq i<j \leq 2$. Taking into account that $i \notin \tau(i)$ for $i \in\{0,1,2\}$, we see that $\tau(i)$ is a singleton for every $i$ and is in fact one of the two possible cyclic shifts on $\{0,1,2\}$. In other words, either $\tau(i)=i+1(\bmod 3)$ for every $i$ or $\tau(i)=i+2(\bmod 3)$ for every $i$. If $\tau(i)=i+1(\bmod 3)$ for every $i$, then $r_{i+2} \notin H_{i}$ for every $i$. This means, that the $i$-th barycentric coordinate of $r_{i+2}$ with respect to $Q$ is strictly negative. This barycentric coordinate is $x_{i+1}+x_{i+2}-1$ since

$$
\begin{aligned}
r_{i+2} & =-p_{i+2}+p_{i}+p_{i+1} \\
& =-\left(\left(1-x_{i+2}\right) q_{i}+x_{i+2} q_{i+1}\right)+\left(\left(1-x_{i}\right) q_{i+1}+x_{i} q_{i+2}\right)+\left(\left(1-x_{i+1}\right) q_{i+2}+x_{i+1} q_{i}\right) \\
& =\left(x_{i+1}+x_{i+2}-1\right) q_{i}+\left(1-x_{i}+x_{i+2}\right) q_{i+1}+\left(1-x_{i+1}+x_{i}\right) q_{i+2} .
\end{aligned}
$$

Thus, we obtain (b). If $\tau(i)=i+2(\bmod 3)$ for every $i$, arguing in the same way we obtain (a).

For proving the converse, we assume that (a) or (b) is fulfilled and show that $Q$ is a lattice-free triangle of type 3 . Consider an arbitrary $p \in \mathbb{Z}^{2}$. We can represent $p$ by $p=z_{0} p_{0}+z_{1} p_{1}+z_{2} p_{2}$ where $z_{i} \in \mathbb{Z}$ and $z_{0}+z_{1}+z_{2}=1$. From symmetry reasons, we may assume that $z_{0} \leq z_{1} \leq z_{2}$. Under these assumptions, we have $z_{2} \geq 1$ and $z_{0} \leq 0$. We evaluate the barycentric coordinates of $p$ with respect to $Q$ as follows:

$$
\begin{aligned}
p & =z_{0} p_{0}+z_{1} p_{1}+z_{2} p_{2} \\
& =z_{0}\left(\left(1-x_{0}\right) q_{1}+x_{0} q_{2}\right)+z_{1}\left(\left(1-x_{1}\right) q_{2}+x_{1} q_{0}\right)+z_{2}\left(\left(1-x_{2}\right) q_{0}+x_{2} q_{1}\right) \\
& =\left(z_{1} x_{1}+z_{2}\left(1-x_{2}\right)\right) q_{0}+\left(z_{0}\left(1-x_{0}\right)+z_{2} x_{2}\right) q_{1}+\left(z_{0} x_{0}+z_{1}\left(1-x_{1}\right)\right) q_{2} .
\end{aligned}
$$

If $z_{1} \leq 0$, then the barycentric coordinate $z_{0} x_{0}+z_{1}\left(1-x_{1}\right)$ is non-positive and by this, in view of Lemma 4.1I, $p \notin$ int $Q$. Assume that $z_{1} \geq 1$. If (a) is fulfilled, then the barycentric coordinate $z_{0} x_{0}+$ $z_{1}\left(1-x_{1}\right)$ is estimated as follows:

$$
z_{0} x_{0}+z_{1}\left(1-x_{1}\right)<z_{0} x_{0}+z_{1} x_{0}=\left(z_{0}+z_{1}\right) x_{0}=\left(1-z_{2}\right) x_{0} \leq 0 .
$$

Consequently, $p \notin \operatorname{int} Q$. If (b) is fulfilled, the barycentric coordinate $z_{0}\left(1-x_{0}\right)+z_{2} x_{2}$ can be estimated analogously:

$$
z_{0}\left(1-x_{0}\right)+z_{2} x_{2}<z_{0}\left(1-x_{0}\right)+z_{2}\left(1-x_{0}\right)=\left(z_{0}+z_{2}\right)\left(1-x_{0}\right)=\left(1-z_{1}\right)\left(1-x_{0}\right) \leq 0 .
$$

Thus, also in this case $p \notin \operatorname{int} Q$. This shows the first part of the lemma. 
We now show the second part. In view of Lemma 4.2 it suffices to show

$$
g(x):=\min \left\{\max _{i=0,1,2}\left|x_{i} y_{i}+\left(1-x_{i+1}\right) y_{i+1}\right|: y \in \mathbb{Z}^{3} \backslash\{o\}, y_{0}+y_{1}+y_{2}=0\right\}=\min \left\{x_{0}, x_{1}, x_{2}\right\}
$$

under the assumption that $x_{i}+x_{j}>1$ for all $0 \leq i<j \leq 2$. Taking all six choices of $y \in\{-1,0,1\}^{3}$ with $y_{0}+y_{1}+y_{2}=0$ we easily verify that

$$
g(x) \leq \min _{i=0,1,2} \max \left\{x_{i+1}, 1-x_{i}\right\}=\min \left\{x_{0}, x_{1}, x_{2}\right\}
$$

where the last inequality is due to assumption (a). It remains to show the converse inequality. Consider $y \in \mathbb{Z}^{3} \backslash\{o\}$ with $y_{0}+y_{1}+y_{2}=0$. Possibly, interchanging $y$ by $-y$, we assure the existence of $j$ such that $y_{j} \geq 0$ and $y_{j+1} \geq 0$. If $y_{j} \geq 1$ and $y_{j+1} \geq 1$, then

$$
\max _{i=0,1,2}\left|x_{i} y_{i}+\left(1-x_{i+1}\right) y_{i+1}\right| \geq x_{j}+1-x_{j+1} \geq x_{j} \geq \min \left\{x_{0}, x_{1}, x_{2}\right\}
$$

Otherwise, one of the $y_{i}$ 's is equal to zero and the remaining ones are equal to $k$ and $-k$ for some $k \in \mathbb{N}$. The latter means we can replace $y$ by $\frac{1}{k} y$, which would decrease $\max \left\{\left|x_{i} y_{i}+\left(1-x_{i+1}\right) y_{i+1}\right|: i=0,1,2\right\}$. We thus arrive at the case $y \in\{-1,0,1\}^{3}$ which has already been considered above.

Let us prove the third part of the lemma. Without loss of generality let $x_{0} \leq x_{1} \leq x_{2}$.

Case 1: $x_{0} \leq \frac{1}{2}$. Then $x_{1}>\frac{1}{2}$ and $x_{2}>\frac{1}{2}$ and we have

$$
\begin{aligned}
\frac{1}{w(Q)} & =\frac{x_{0} x_{1} x_{2}+\left(1-x_{0}\right)\left(1-x_{1}\right)\left(1-x_{2}\right)}{x_{0}} \\
& \geq \frac{x_{0} x_{1} x_{2}+x_{0}\left(1-x_{1}\right)\left(1-x_{2}\right)}{x_{0}} \\
& =\frac{1}{2}\left(2 x_{1}-1\right)\left(2 x_{2}-1\right)+\frac{1}{2}>\frac{1}{2},
\end{aligned}
$$

which implies that $w(Q)<2$.

Case 2: $x_{0}>\frac{1}{2}$. We use the notations $\sigma_{1}(x):=x_{0}+x_{1}+x_{2}$ and $\sigma_{2}(x):=x_{0} x_{1}+x_{0} x_{2}+x_{1} x_{2}$. Clearly, $2 \sigma_{2}(x)-\sigma_{1}(x)=\left(x_{0}+x_{1}-1\right) x_{2}+\left(x_{0}+x_{2}-1\right) x_{1}+\left(x_{1}+x_{2}-1\right) x_{0} \geq\left(2\left(x_{0}+x_{1}+x_{2}\right)-3\right) x_{0}=\left(2 \sigma_{1}(x)-3\right) x_{0}$ and by this

$$
\begin{aligned}
x_{0} x_{1} x_{2}+\left(1-x_{0}\right)\left(1-x_{1}\right)\left(1-x_{2}\right) & =1-\sigma_{1}(x)+\sigma_{2}(x) \geq 1-\frac{1}{2} \sigma_{1}(x)+\left(\sigma_{1}(x)-\frac{3}{2}\right) x_{0} \\
& =1-\frac{3}{2} x_{0}+\left(x_{0}-\frac{1}{2}\right) \sigma_{1}(x) \\
& \geq 1-\frac{3}{2} x_{0}+\left(x_{0}-\frac{1}{2}\right) 3 x_{0}=1-3 x_{0}+3 x_{0}^{2} .
\end{aligned}
$$

Consequently, applying elementary calculus, we get

$$
\frac{1}{w(Q)} \geq 3 x_{0}-3+\frac{1}{x_{0}} \geq 2 \sqrt{3}-3
$$

which implies $w(Q) \leq 1+\frac{2}{\sqrt{3}}$ and shows that the equality $w(Q)=1+\frac{2}{\sqrt{3}}$ is attained if and only if $x_{0}=x_{1}=x_{2}=\frac{1}{\sqrt{3}}$. 
The following two lemmas prepare the proof of Theorem 2.2 . Parts of the proof of Lemma 5.2 are borrowed from [10; nevertheless we need these parts for subsequent arguments.

Lemma 5.2. Let $K \in \mathcal{K}^{2}$ be maximal lattice-free with $[0,1]^{2} \subseteq K$ and let $w:=w(K)$ and $A:=A(K)$. Then $w \leq 2$ and either it holds $w=1$ and $A=\infty$ (i.e. $K$ is a split) or $w>1$ and $A \leq \frac{w^{2}}{2(w-1)}$ with equality $A=\frac{w^{2}}{2(w-1)}$ characterized by Part II of Theorem 2.2.

Proof. If $w=1$ there is nothing to show. Thus, assume $w>1$ and therefore $K$ is a triangle of type 1 , type 2 or a quadrilateral. We only consider the case that $K$ is a quadrilateral. The case where $K$ is a triangle can be viewed as a degenerate version of a quadrilateral where one vertex becomes a convex combination of its two neighbor vertices. By $a_{1}, a_{2}, a_{3}, a_{4}$ we denote the consecutive vertices of $[0,1]^{2}$. Let $q_{1}, q_{2}, q_{3}, q_{4}$ be consecutive vertices of $K$ such that the point $q_{i}^{\prime}$ of $[0,1]^{2}$ closest to $q_{i} \operatorname{lies}$ in $\operatorname{conv}\left\{a_{i}, a_{i+1}\right\}$. The distance from $q_{i}$ to $q_{i}^{\prime}$ will be denoted by $h_{i}$ and the distance from $a_{i}$ to $q_{i}^{\prime}$ by $t_{i}$, see also Fig. 8 . Taking into account the relations $h_{i} h_{i-1}=t_{i}\left(1-t_{i-1}\right)$ it can be verified that

$$
\begin{aligned}
& 1-\left(h_{1}+h_{3}\right)\left(h_{2}+h_{4}\right) \\
= & \left(1-t_{1}-t_{3}\right)\left(1-t_{2}-t_{4}\right) \\
= & \frac{\left(h_{1} h_{2} h_{3} h_{4}-t_{1} t_{2} t_{3} t_{4}\right)^{2}}{t_{1} t_{2} t_{3} t_{4}} \geq 0,
\end{aligned}
$$

for details see [10, p. 124]. Without loss of generality we assume that $h_{1}+h_{3} \leq h_{2}+h_{4}$. Relations (5.3) yield $h_{1}+h_{3} \leq 1$. Thus, the width of $K$ with respect to the vector $u=(1,0)$ is $h_{1}+h_{3}+1 \leq 2$. For all vectors $u \in \mathbb{Z}^{2} \backslash\{o\}$ which are not in $\{ \pm(1,0), \pm(0,1)\}$ we easily get $w(K, u) \geq w\left([0,1]^{2}, u\right) \geq 2$. Hence $w=h_{1}+h_{3}+1 \leq 2$. Furthermore,

$$
\begin{aligned}
A & =1+\frac{1}{2}\left(h_{1}+h_{2}+h_{3}+h_{4}\right)=1+\frac{1}{2}\left(w-1+h_{2}+h_{4}\right) \\
& \leq 1+\frac{1}{2}\left(w-1+\frac{1}{h_{1}+h_{3}}\right)=1+\frac{1}{2}\left(w-1+\frac{1}{w-1}\right)=\frac{w^{2}}{2(w-1)} .
\end{aligned}
$$

If the equality $A=\frac{w^{2}}{2(w-1)}$ is attained, then $h_{2}+h_{4}+1=\frac{w}{w-1}$ and $\left(1-t_{1}-t_{3}\right)\left(1-t_{2}-t_{4}\right)=0$, which implies that $1-t_{1}-t_{3}=0$ or $1-t_{2}-t_{4}=0$. Taking into account the geometric meaning of $t_{i}$ 's, we see (using (5.3) ) that the equalities $1-t_{1}-t_{3}=0$ and $1-t_{2}-t_{4}=0$ imply one another so that one has $1-t_{1}-t_{3}=1-t_{2}-t_{4}=0$. The above relations yield the characterization of the equality case given in Part II of Theorem 2.2.

Lemma 5.3. Let $K \in \mathcal{K}^{2}$ be a maximal lattice-free triangle with $w:=w(K)$ and $A:=A(K)$. Then $w>1$ and (2.3) resp. (2.4) holds true. The equality case in both inequalities is characterized by Part II resp. Part III of Theorem 2.2.

Proof. If $K$ is a triangle of type 1 or type 2 , then clearly $w>1$. Consider a triangle $K$ of type 3 and a set $P=\operatorname{conv}\left\{p_{0}, p_{1}, p_{2}\right\}$, as in Lemma 5.1 such that the relative interior of each edge of $K$ contains a point from $\left\{p_{0}, p_{1}, p_{2}\right\}$. Then, for every vector, the width function of $K$ is strictly larger than that of $P$, and furthermore, the lattice width of $P$ is equal to 1 . It follows that $w>1$.

If $K$ contains more than three integer points (and thus there is a unimodular transformation of $K$ which contains $[0,1]^{2}$ ), the assumptions of Lemma 5.2 are fulfilled and the assertion follows directly from Lemma 5.2 . 


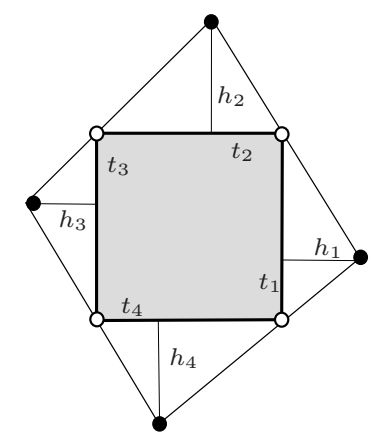

Figure 8: A maximal lattice-free quadrilateral in the proof of Theorem 2.2

Now assume that every edge of $K$ contains precisely one integer point, i.e., $K$ is a triangle of type 3 . We define $K=Q=\operatorname{conv}\left\{q_{0}, q_{1}, q_{2}\right\}$ with $q_{0}, q_{1}, q_{2}$ and $Q$ given as in Lemma 5.1 and also borrow the other notations of Lemma 5.1. Without loss of generality we assume that $x_{0} \leq x_{1} \leq x_{2}$ and $x_{i}+x_{j}>1$ for all $0 \leq i<j \leq 2$. Let $f(x):=x_{0} x_{1} x_{2}+\left(1-x_{0}\right)\left(1-x_{1}\right)\left(1-x_{2}\right)$. The upper bound for $w$ follows from Lemma 5.1

Case 1: $x_{0} \geq \frac{1}{2}$. If $1<w<2$, then in view of Lemma 4.2 we obtain

$$
A=\frac{1}{2 f(x)}=\frac{x_{0}}{f(x)} \cdot \frac{1}{2 x_{0}}=\frac{w}{2 x_{0}} \leq w<\frac{w^{2}}{2(w-1)} .
$$

Assume now that $w \geq 2$. Then, taking into account Lemmas 5.1 and 4.2 we obtain

$$
\begin{aligned}
A & \leq \max \left\{\frac{1}{2 f(x)}: \frac{1}{2} \leq x_{0} \leq x_{1} \leq x_{2}<1, x_{0}=w f(x)\right\} \\
& =\frac{1}{2}\left(\min \left\{f(x): \frac{1}{2} \leq x_{0} \leq x_{1} \leq x_{2}<1, x_{0}=w f(x)\right\}\right)^{-1} .
\end{aligned}
$$

Furthermore, using (5.2), we obtain

$$
\begin{aligned}
& \min \left\{f(x): \frac{1}{2} \leq x_{0} \leq x_{1} \leq x_{2}<1, x_{0}=w f(x)\right\} \\
= & \frac{1}{w} \min \left\{x_{0}: \frac{1}{2} \leq x_{0} \leq x_{1} \leq x_{2}<1, x_{0}=w f(x)\right\} \\
\geq & \frac{1}{w} \min \left\{x_{0}: \frac{1}{2} \leq x_{0}<1, x_{0} \geq w\left(1-3 x_{0}+3 x_{0}^{2}\right)\right\} .
\end{aligned}
$$

The minimum in the previous expression is attained for $x_{0}$ equal to the smaller root of the equation $t=w\left(1-3 t+3 t^{2}\right)$ since this root, which is equal to

$$
\frac{3 w+1-\sqrt{1+6 w-3 w^{2}}}{6 w}
$$

lies in $\left[\frac{1}{2}, 1\right)$. The characterization of the equality case follows directly by analyzing the equality cases in the above estimates. Thus, equality holds for $x_{0}=x_{1}=x_{2}$. 
Case 2: $x_{0}<\frac{1}{2}$. We have

$$
\begin{aligned}
I & :=\inf \left\{f(x): 0<x_{0}<\frac{1}{2}, \frac{1}{2}<x_{1} \leq x_{2}<1, x_{0}+x_{1}>1, x_{0}+x_{2}>1, x_{0}=w f(x)\right\} \\
& =\frac{1}{w} \inf \left\{x_{0}: 0<x_{0}<\frac{1}{2}, \frac{1}{2}<x_{1} \leq x_{2}<1, x_{0}+x_{1}>1, x_{0}+x_{2}>1, x_{0}=w f(x)\right\} \\
& \geq \frac{1}{w} \inf \left\{x_{0}: 0<x_{0}<\frac{1}{2}, \frac{1}{2}<x_{1} \leq x_{2}<1, x_{0}+x_{1}>1, x_{0}+x_{2}>1, x_{0} \geq w f(x)\right\} .
\end{aligned}
$$

Furthermore,

$$
\begin{aligned}
f(x) & =\left(x_{0}+x_{1}-1\right) x_{2}+\left(1-x_{0}\right)\left(1-x_{1}\right) \\
& \geq\left(x_{0}+x_{1}-1\right) x_{1}+\left(1-x_{0}\right)\left(1-x_{1}\right) \\
& =x_{0}\left(2 x_{1}-1\right)+\left(1-x_{1}\right)^{2} \\
& =\left(1-x_{0}\right) x_{0}+\left(x_{0}+x_{1}-1\right)^{2} \\
& >\left(1-x_{0}\right) x_{0} .
\end{aligned}
$$

Hence

$$
\begin{aligned}
I & \geq \frac{1}{w} \inf \left\{x_{0}: 0<x_{0}<\frac{1}{2}, x_{0}>w x_{0}\left(1-x_{0}\right)\right\} \\
& =\frac{1}{w} \inf \left\{x_{0}: 1-\frac{1}{w}<x_{0}<\frac{1}{2}\right\} \\
& =\frac{1}{w}\left(1-\frac{1}{w}\right) .
\end{aligned}
$$

It follows that

$$
A=\frac{1}{2 f(x)} \leq \frac{1}{2 I} \leq \frac{w^{2}}{2(w-1)} .
$$

The equality case is characterized in a straightforward way.

We notice that Theorem 2.1 is a consequence of Propositions 3.2, 3.1 and Lemmas 5.2, 5.3 (in fact, the main steps of the proof from [10] were incorporated in Lemmas 5.1] 5.2 and [5.3).

Proof of Theorem 2.2. Let us show (2.2)-(2.4). In view of Lemmas 5.2, 5.3 bounds (2.2)-(2.4) hold for maximal lattice-free sets. Let $K \in \mathcal{K}^{2}$ be an arbitrary lattice-free set. By Proposition [3.1] there exists a maximal lattice-free set $H \in \mathcal{K}^{2}$ such that $K \subseteq H$. We have $w(K) \leq w(H)$ and $A(K) \leq A(H)$. For $0<w \leq 1+\frac{2}{\sqrt{3}}$ define $F(w)$ to be the upper bounds in (2.2)-(2.4). Note that $F(w)$ is monotonically non-increasing. Thus, it follows $A(K) \leq A(H) \leq F(w(H)) \leq F(w(K))$. The equality $A(K)=F(w(K))$ implies $K=H$ and, thus, the characterizations of the equality cases for (2.2)-(2.4) follow from the characterizations of the equality cases in Lemmas 5.2, 5.3. The bound (2.5) and Part IV follow directly from Theorem 2.3 .

Proof of Corollary 2.6. We have $\mu_{1}(K) w(K)=1$. Furthermore, an appropriate translate of $\mu_{2}(K) \cdot K$ is lattice-free. Thus, we apply the upper bounds of Theorem 2.2 to the body $\mu_{2}(K) \cdot K$ and then express the lattice width of $\mu_{2}(K) \cdot K$ as $\frac{\mu_{2}(K)}{\mu_{1}(K)}$. The sharpness of the bounds follows from the characterizations of the equality cases in Theorem 2.2 


\section{Proofs for centrally symmetric bodies}

In this section we prove Theorems 2.4 and 2.5. Inequalities (2.6) have already been stated in 12 , Theorem 2.13], but the proof of Kannan and Lovász does not seem to show this result. Therefore, we first show (2.6) by proving Theorem 2.5. Afterwards, we use (2.6) to show (2.7)-(2.9).

Proof of Theorem 2.5. We want to show that $\mu_{2}(K) \leq 2 \mu_{1}(K)$ for every centrally symmetric $K \in \mathcal{K}^{2}$. For convenience we define $\mu_{i}:=\mu_{i}(K)$ for $i=1,2$ and $w:=w(K)$. We further assume that $K$ has $o$ as its center of symmetry. Since both $\mu_{1}$ and $\mu_{2}$ are homogeneous of degree -1 , it suffices to consider the case $\mu_{2}=1$. Using $\mu_{1} w=1$ it remains to prove that $w \leq 2$.

In this proof, when we say hexagon we refer to a centrally symmetric convex hexagon which could also be degenerated so as to be a parallelogram. A hexagon will be given by a sequence of consecutive vertices indexed modulo 6. Because of degeneracy we allow the "vertices" to be collinear or to coincide.

Since $\mu_{2}=1$, the translates $K+z$ with $z \in \mathbb{Z}^{2}$ cover $\mathbb{R}^{2}$. By [8, Lemma 3, p. 246] there exists a hexagon $Q:=\operatorname{conv}\left\{q_{i}: i=0, \ldots, 5\right\}$ centered at $o$ such that the vertices of $Q$ lie on the boundary of $K$ and the translates $Q+z$ with $z \in \mathbb{Z}^{2}$ tile $\mathbb{R}^{2}$. We introduce a second hexagon $R:=\operatorname{conv}\left\{r_{i}: i=0, \ldots, 5\right\}$, centered at $o$ and containing $Q$, such that for every $i$ the line aff $\left\{r_{i}, r_{i+1}\right\}$ supports $K$ at $q_{i}$. This implies $\mu_{2}(R)=1$ and from $K \subseteq R$ it follows that $w \leq w(R)$. Thus, it suffices to bound $w(R)$ from above.

Let $P:=\operatorname{conv}\left\{p_{i}: i=0, \ldots, 5\right\}$ be the hexagon with $p_{i}:=\frac{1}{2}\left(q_{i}+q_{i+1}\right)$ for every $i$. From the choice of $Q$ it follows that $2 p_{i}, 2 p_{i+1}$ is a basis of $\mathbb{Z}^{2}$ for every $i$. Thus, we constructed hexagons $P \subseteq Q \subseteq R$ such that every vertex of $P$ is the central point of an edge of $Q$, and each edge of $R$ contains a vertex of $Q$ (see Fig. $9(\mathrm{a})$. We show that for such a configuration there exists an edge of $P$ such that its normal, say $u$, satisfies $w(R, u) \leq 2 w(P, u)$. In terms of lattices this means that $w(R) \leq 2$, which then finishes the proof.

By $u_{i}$ we shall denote the normal vector to the edge $\operatorname{conv}\left\{p_{i}, p_{i+1}\right\}$ of $P$. Let us take any vertex $r_{i}$ of $R$ such that some line through $r_{i}$ which is parallel to an edge of $P$ supports $R$. This supporting line is then parallel to either aff $\left\{p_{i-1}, p_{i}\right\}$ or aff $\left\{p_{i-2}, p_{i-1}\right\}$. By symmetry we may assume that the line through $r_{i}$ parallel to aff $\left\{p_{i-1}, p_{i}\right\}$ supports $R$. Now consider the parallelogram $R^{\prime}$ which is the intersection of the four halfplanes defined by the lines \pm aff $\left\{q_{i-1}, q_{i}\right\}$ and \pm aff $\left\{r_{i+1}, r_{i+2}\right\}$ containing $o$. It can be seen that $w\left(R, u_{j}\right) \leq w\left(R^{\prime}, u_{j}\right)$ for every $j$. Thus, we can replace $R$ by $R^{\prime}$ and it suffices to show $w\left(R^{\prime}\right) \leq 2$.

The edge $\operatorname{conv}\left\{q_{i-1}, q_{i}\right\}$ of $Q$ lies on the boundary of $R^{\prime}$. We modify $Q$ and $R^{\prime}$ simultaneously to obtain $Q^{\prime}$ and $R^{\prime \prime}$, respectively, in the following way. Define $Q^{\prime}:=\operatorname{conv}\left\{ \pm p_{i-1}, \pm\left(p_{i}+p_{i+1}\right)\right\}$ and define $R^{\prime \prime}$ as the parallelogram circumscribing $Q^{\prime}$ with edges parallel to the edges of $R^{\prime}$ (see Fig. 9(b)). Clearly, $R^{\prime} \subseteq R^{\prime \prime}$ and by this $w\left(R^{\prime}, u_{j}\right) \leq w\left(R^{\prime \prime}, u_{j}\right)$ for every $j$.

With our modifications we have reduced the problem on hexagons to a problem on quadrilaterals, i.e., where both $Q$ and $R$ are quadrilaterals. Now, the assertion follows using the same arguments as in the proof of Lemma 5.2 There is a unimodular transformation which maps $Q$ to $[0,1]^{2}$ and $R$ to a quadrilateral $K=\operatorname{conv}\left\{q_{1}, q_{2}, q_{3}, q_{4}\right\}$ as in Lemma 5.2 (see also Fig. 8), where we have showed that $w(K, u) \leq 2$ for $u=(1,0)$ or $u=(0,1)$.

Remember that every o-symmetric $K \in \mathcal{K}^{2}$ can be associated with a norm

$$
\|u\|_{K}=\min \{\alpha \geq 0: u \in \alpha K\} .
$$

It is well known that $\|u\|_{K}=h\left(K^{*}, u\right)$.

Lemma 6.1. Let $0 \leq \alpha<1$ and let $K_{\alpha}:=\operatorname{conv}\{ \pm(1, \alpha), \pm(0,1)\}$. Then $\mu_{2}\left(K_{\alpha}\right)=\frac{1}{2} \max \{1+\alpha, 2-\alpha\}$. 


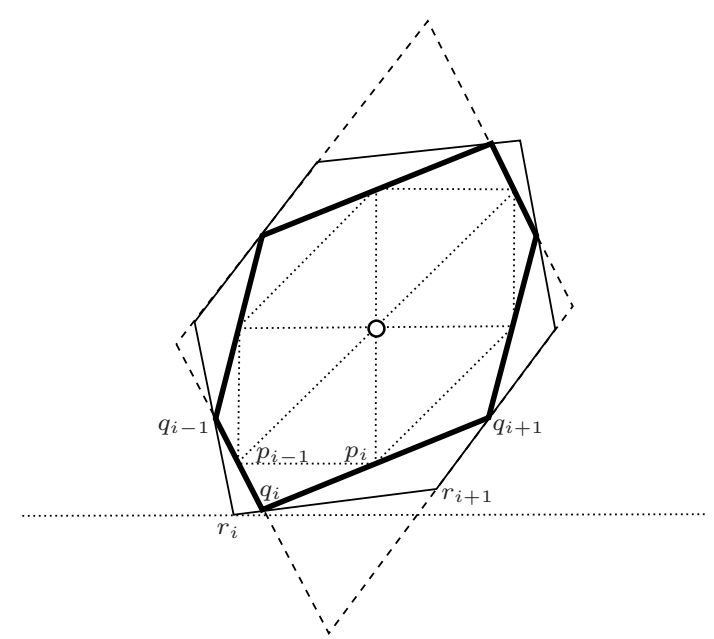

(a) Reduction of $R$ (thin solid) to a parallelogram $R^{\prime}$ (dashed); $R$ and $R^{\prime}$ are circumscribed about $Q$ (bold solid); $Q$ is circumscribed about $P$ (dotted)

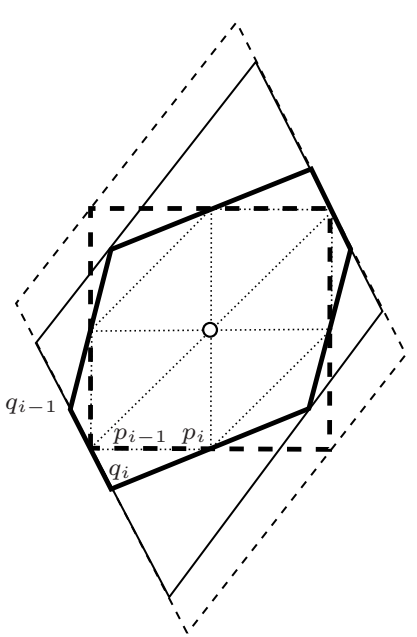

(b) Reduction of $Q$ (bold solid) and $R^{\prime}$ (thin solid) to parallelograms $Q^{\prime}$ (bold dashed) and $R^{\prime \prime}$ (thin dashed), respectively

Figure 9: The hexagons $Q$ and $R$ degenerate into parallelograms

Proof. The unimodular transformation given by the matrix $\left(\begin{array}{cc}1 & 0 \\ 1 & -1\end{array}\right)$ maps $K_{\alpha}$ onto $K_{1-\alpha}$. Thus, it suffices to consider the case $0 \leq \alpha \leq \frac{1}{2}$. For the sake of brevity we write $K:=K_{\alpha}$. Direct computations show that $K^{*}=\operatorname{conv}\{ \pm(-\alpha+1,1), \pm(-\alpha-1,1)\}=\operatorname{conv}\{(-\alpha, 1),(\alpha,-1)\}+\operatorname{conv}\{(1,0),(-1,0)\}$. Hence

$$
f(u):=h\left(K^{*}, u\right)=\left|u_{2}-\alpha u_{1}\right|+\left|u_{1}\right|,
$$

for $u=\left(u_{1}, u_{2}\right) \in \mathbb{R}^{2}$. We have

$$
\begin{aligned}
\mu_{2}(K) & =\min \left\{\mu \geq 0: \mu K+\mathbb{Z}^{2}=\mathbb{R}^{2}\right\} \\
& =\min \left\{\mu \geq 0: \forall x \in \mathbb{R}^{2} \exists z \in \mathbb{Z}^{2} \text { such that }\|x-z\|_{K} \leq \mu\right\} \\
& =\max _{x \in \mathbb{R}^{2}} \min _{z \in \mathbb{Z}^{2}}\|x-z\|_{K} \\
& =\max _{x \in \mathbb{R}^{2}} \min _{z \in \mathbb{Z}^{2}} h\left(K^{*}, x-z\right) \\
& =\max _{x \in \mathbb{R}^{2}} \min _{z \in \mathbb{Z}^{2}} f(x-z) .
\end{aligned}
$$

For $t \in \mathbb{R}$ let $\lfloor t\rceil$ denote the nearest integer function. If $s, t \in \mathbb{R}$, we introduce the distance between $s$ and $t$ modulo 1 by $d(s, t):=d(s-t, 0)$, where $d(s, 0):=|s-\lfloor s\rceil|$. We choose $x=\left(x_{1}, x_{2}\right) \in \mathbb{R}^{2}$ and consider

$$
f(x-z)=\left|x_{2}-z_{2}-\alpha\left(x_{1}-z_{1}\right)\right|+\left|x_{1}-z_{1}\right| .
$$

with $z=\left(z_{1}, z_{2}\right)$ varying in $\mathbb{Z}^{2}$. For $z_{1}=\left\lfloor x_{1}\right\rceil$ and $z_{2}=\left\lfloor x_{2}-\alpha\left(x_{1}-z_{1}\right)\right\rceil$ we have $f(x-z) \leq 1$. Furthermore, if $z_{1} \notin\left\{\left\lfloor x_{1}\right\rfloor,\left\lceil x_{1}\right\rceil\right\}$, then $f(x-z) \geq\left|x_{1}-z_{1}\right| \geq 1$. Thus, computing (6.1) we may assume that $z_{1} \in\left\{\left\lfloor x_{1}\right\rfloor,\left\lceil x_{1}\right\rceil\right\}$. If $x_{1} \in \mathbb{Z}$ we may set $z_{1}=x_{1}$ and $z_{2}=\left\lfloor x_{2}\right\rceil$ obtaining $f(x-z) \leq \frac{1}{2}$. 
Otherwise $x_{1} \in \mathbb{R} \backslash \mathbb{Z}$ and we introduce $\beta:=x_{1}-\left\lfloor x_{1}\right\rfloor$ satisfying $0<\beta<1$. Since we can assume that $z_{1} \in\left\{\left\lfloor x_{1}\right\rfloor,\left\lceil x_{1}\right\rceil\right\}$ we have

$$
\begin{aligned}
\min _{z \in \mathbb{Z}^{2}} f(x-z) & =\min _{z_{2} \in \mathbb{Z}} \min \left\{\left|x_{2}-z_{2}-\alpha \beta\right|+\beta,\left|x_{2}-z_{2}+\alpha(1-\beta)\right|+1-\beta\right\} \\
& =\min \left\{d\left(x_{2}-\alpha \beta, 0\right)+\beta, d\left(x_{2}+\alpha(1-\beta), 0\right)+1-\beta\right\} .
\end{aligned}
$$

Thus, the value in (6.1) is the maximum of $\frac{1}{2}$ and the value

$$
\begin{aligned}
\max _{\substack{x \in \mathbb{R}^{2} \\
x_{1} \notin \mathbb{Z}}} \min _{z \in \mathbb{Z}^{2}} f(x-z) & =\max _{\substack{0<\beta<1 \\
x_{2} \in \mathbb{R}}} \min \left\{d\left(x_{2}-\alpha \beta, 0\right)+\beta, d\left(x_{2}+\alpha(1-\beta), 0\right)+1-\beta\right\} \\
& =\max _{\substack{0<\beta<1 \\
x_{2} \in \mathbb{R}}} \min \left\{d\left(x_{2}+\alpha(1-\beta), \alpha\right)+\beta, d\left(x_{2}+\alpha(1-\beta), 0\right)+1-\beta\right\} \\
& =\max _{\substack{0<\beta<1 \\
x_{2} \in \mathbb{R}}} \min \left\{d\left(x_{2}, \alpha\right)+\beta, d\left(x_{2}, 0\right)+1-\beta\right\} \\
& \leq \max _{\beta, x_{2} \in \mathbb{R}} \min \left\{d\left(x_{2}, \alpha\right)+\beta, d\left(x_{2}, 0\right)+1-\beta\right\}
\end{aligned}
$$

If $d\left(x_{2}, \alpha\right)+\beta$ and $d\left(x_{2}, 0\right)+1-\beta$ differ, then sligthly perturbing $\beta$ the minimum of these two values becomes larger. Hence the latter maximum is attained for the $\beta$ for which $d\left(x_{2}, \alpha\right)+\beta$ and $d\left(x_{2}, 0\right)+1-\beta$ coincide. In this case $\beta=\frac{1}{2}\left(d\left(x_{2}, 0\right)-d\left(x_{2}, \alpha\right)+1\right)$. Thus

$$
\begin{aligned}
\max _{\beta, x_{2} \in \mathbb{R}} \min \left\{d\left(x_{2}, \alpha\right)+\beta, d\left(x_{2}, 0\right)+1-\beta\right\} & =\max _{x_{2} \in \mathbb{R}} \frac{1}{2}\left(d\left(x_{2}, 0\right)+d\left(x_{2}, \alpha\right)+1\right) \\
& =\frac{1}{2}\left(1+\max _{x_{2} \in \mathbb{R}}\left(d\left(x_{2}, 0\right)+d\left(x_{2}, \alpha\right)\right)\right) \\
& =\frac{1}{2}(2-\alpha) .
\end{aligned}
$$

Since the latter is at least $\frac{1}{2}$ we have shown $\mu_{2}(K) \leq \frac{1}{2}(2-\alpha)$. It remains to show that the above is attained with equality. Employing the above derivations we can see that the equality is attained for $x_{1}=\frac{1+\alpha}{2}$ and $x_{2}=\frac{1}{2}$.

Proof of Theorem 2.4. Since (2.6) is established the bounds (2.7) and (2.8) together with Parts II] and III follow directly from Theorem 2.2 .

Now let us show (2.9) and Part IV. By (3.1), $o$ is the only interior integer point in $w \cdot(D K)^{*}$, where $w:=w(K)$. Thus, by Minkowski's first theorem, $A\left(w \cdot(D K)^{*}\right) \leq 4$. Using the above fact and Mahler's inequality we obtain

$$
A(K)=\frac{A(D K)}{4}=\frac{A(D K) A\left((D K)^{*}\right)}{4 A\left((D K)^{*}\right)} \geq \frac{2}{A\left((D K)^{*}\right)} \geq \frac{w^{2}}{2} .
$$

It remains to characterize the case where this inequality is tight. In view of Mahler's inequality and Minkowski's first theorem (the parts in these statements which give information on the equality cases), the equality $A(K)=\frac{w^{2}}{2}$ implies that $(D K)^{*}$ is a parallelogram and that the sets $\frac{w}{2}(D K)^{*}+z$ with $z \in \mathbb{Z}^{2}$ tile $\mathbb{R}^{2}$. By Proposition 3.3 we deduce that there exists a linear unimodular transformation $T$ and a parameter $0 \leq \alpha<1$ such that

$$
T\left(\frac{w}{2}(D K)^{*}\right)=\frac{1}{2} \operatorname{conv}\{ \pm(-\alpha-1,1), \pm(-\alpha+1,1)\}
$$


Taking duals and slightly modifying the left hand side of the equality we arrive at

$$
\frac{2}{w}\left(T^{-1}\right)^{*}(D K)=2(\operatorname{conv}\{ \pm(-\alpha-1,1), \pm(-\alpha+1,1)\})^{*} .
$$

Direct computations yield

$$
(\operatorname{conv}\{ \pm(-\alpha-1,1), \pm(-\alpha+1,1)\})^{*}=\operatorname{conv}\{ \pm(1, \alpha), \pm(0,1)\} .
$$

Clearly, the transformation $\left(T^{-1}\right)^{*}$ is unimodular. Summarizing we see that, up to unimodular transformations, we have

$$
\frac{1}{2} D K=\frac{w}{2} \operatorname{conv}\{ \pm(1, \alpha), \pm(0,1)\}
$$

with $0 \leq \alpha<1$. Note that $\frac{1}{2} D K$ is a translate of $K$. An appropriate translate of $\frac{1}{2} D K$ is lattice-free if and only if $\mu_{2}\left(\frac{1}{2} D K\right) \geq 1$. In view of Lemma 6.1] we get $\mu_{2}\left(\frac{1}{2} D K\right)=\frac{2}{w} \cdot \frac{1}{2} \max \{1+\alpha, 2-\alpha\}$. The above observations yield Part IV

Proof of Corollary 2.7. The proof is analogous to the proof of Corollary 2.6.

\section{Acknowledgements}

We thank the anonymous referee, E. Makai, Jr. and C. A. J. Hurkens for useful suggestions and pointers to the literature.

\section{References}

[1] G. Averkov, C. Wagner, and R. Weismantel, All three-dimensional maximal lattice-free polytopes with integer vertices, manuscript, 2009.

[2] A. Basu, M. Conforti, G. Cornuéjols, and G. Zambelli, Maximal lattice-free convex sets in linear subspaces, manuscript, 2009.

[3] U. Betke, M. Henk, and J. M. Wills, Successive-minima-type inequalities, Discrete Comput. Geom. 9 (1993), no. 2, 165-175. MR 93j:52026

[4] H. S. M. Coxeter, Introduction to Geometry, Wiley Classics Library, John Wiley \& Sons Inc., New York, 1989, Reprint of the 1969 edition. MR 90a:51001

[5] S. S. Dey and L. A. Wolsey, Two row mixed integer cuts via lifting, Technical Report CORE DP 30, Université catholique de Louvain, Louvain-la-Neuve, Belgium (2008).

[6] L. Fejes Tóth and E. Makai, Jr., On the thinnest non-separable lattice of convex plates, Stud. Sci. Math. Hungar. 9 (1974), 191-193. MR 51 \#6596

[7] P. M. Gruber, Convex and Discrete Geometry, Grundlehren der Mathematischen Wissenschaften, vol. 336, Springer, Berlin, 2007. MR 2008f:52001

[8] P. M. Gruber and C. G. Lekkerkerker, Geometry of Numbers, second ed., North-Holland Mathematical Library, vol. 37, North-Holland Publishing Co., Amsterdam, 1987. MR 88j:11034

[9] G. Hajós, Über einfache und mehrfache Bedeckung des n-dimensionalen Raumes mit einem Würfelgitter, Math. Z. 47 (1941), 427-467, MR 3,302b 
[10] C. A. J. Hurkens, Blowing up convex sets in the plane, Linear Algebra Appl. 134 (1990), 121-128. MR 91i:52009

[11] R. Kannan and L. Lovász, Covering minima and lattice point free convex bodies, Foundations of software technology and theoretical computer science (New Delhi, 1986), Lecture Notes in Comput. Sci., vol. 241, Springer, Berlin, 1986, pp. 193-213. MR 89b:11055

[12] Covering minima and lattice-point-free convex bodies, Ann. of Math. (2) 128 (1988), no. 3, 577-602. MR 89i:52020

[13] A. Ya. Khinchin, A quantitative formulation of the approximation theory of Kronecker, Izv. Akad. Nauk SSSR Ser. Mat. 12 (1948), no. 2, 113-122.

[14] L. Lovász, Geometry of numbers and integer programming, Math. Prog., Recent Developments and Applications, Kluwer Academic Publishers, Dordrecht (1989), 177-201.

[15] K. Mahler, Ein Übertragungsprinzip für lineare Ungleichungen, Časopis Pěst. Mat. Fys. 68 (1939), 85-92. MR 1,202b

[16] E. Makai, Jr., On the thinnest non-separable lattice of convex bodies, Stud. Sci. Math. Hungar. 13 (1978), no. 1-2, 19-27. MR 83a:52016

[17] U. Schnell, A Minkowski-type theorem for covering minima in the plane, Geom. Dedicata 55 (1995), no. 3, 247-255. MR 96f:52020

[18] A. Schrijver, Theory of Linear and Integer Programming, Wiley-Interscience Series in Discrete Mathematics, John Wiley \& Sons Ltd., Chichester, 1986. MR 88m:90090

[19] E. Schulte, Tilings, Handbook of Convex Geometry, vol. A, B, North-Holland, Amsterdam, 1993, pp. 899-932. MR 94g:52026

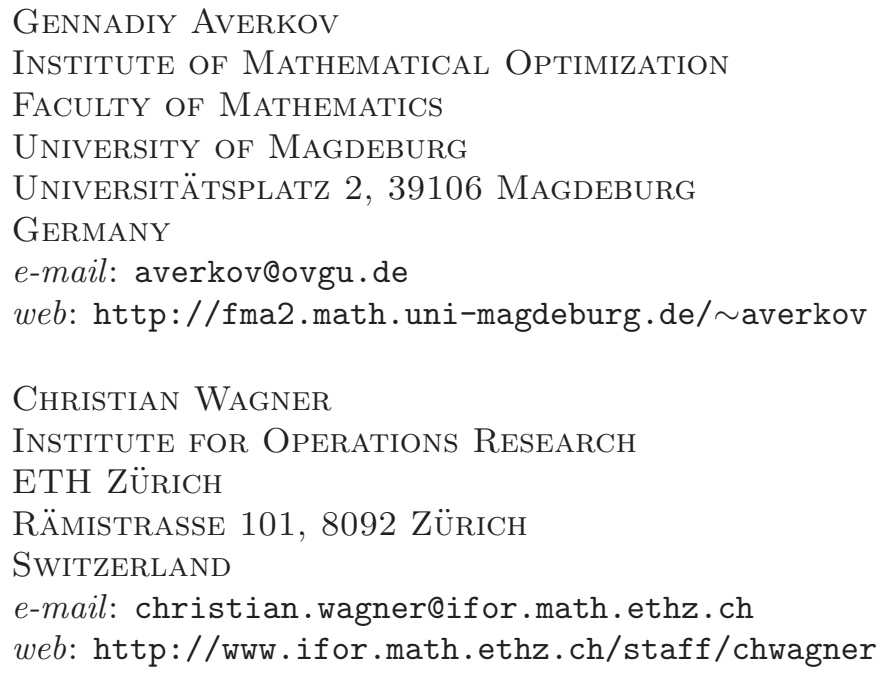

